\title{
Non-Operative Rehabilitation of Isolated Popliteus Tendon Rupture in a Rugby Player
}

\author{
Denis Pak-Han Koong, MD, BMed ${ }^{1}$, Vincent Vinh Gia An, MD, BSci ${ }^{2}$, Peter Lorentzos, MBBS, BSci ${ }^{3}$, \\ Peter Moussa, BAppSc ${ }^{3}$, and Brahman Shankar Sivakumar, MBBS, BSci ${ }^{1}$ \\ ${ }^{1}$ Department of Orthopaedic Surgery, Royal North Shore Hospital, Sydney; ${ }^{2}$ Sydney Medical School, University of Sydney, Sydney; ${ }^{3}$ Wests Tigers Rugby League Football \\ Club, Sydney, Australia
}

\begin{abstract}
Isolated rupture of the popliteus tendon is uncommon; instead, it is often seen as part of multi-ligamentous posterolateral corner injuries. In this report, we present a case of a 22 -year-old professional rugby player who sustained a lateral blow to his semi-flexed knee in a tackle during a competitive game. A complete popliteus tendon rupture at its musculo-tendinous junction was diagnosed on magnetic resonance imaging despite a relatively unremarkable physical examination. The aims of this report are to highlight the diagnostic challenges with this rare injury as physical signs are often subtle and non-specific. Furthermore, we demonstrate the viability of conservative management in the setting of a direct contact mechanism. Indeed, our patient was successfully treated with a specific rehabilitation protocol via isometric quadriceps contractions, gastrocnemiussoleus and hamstring strengthening exercises and graded activity with successful return to full contact activities at 4 weeks and regular season matches shortly after.
\end{abstract}

Keywords: Knee, Popliteus tendon, Injury, Rehabilitation

Often seen as part of a complex multi-ligamentous knee injury, isolated rupture of the popliteus tendon is uncommon ${ }^{1-3)}$. Diagnosis in the acute setting can be easily overlooked but an acute haemarthrosis and posterolateral pain in an otherwise stable knee should raise suspicion of this injury ${ }^{4,5)}$. Currently there is no consensus on the optimal treatment but there is increasing evidence that conservative management results in comparable short- and long-term functional outcomes compared to surgical repair $^{5-8)}$. We present a case of a high-level contact athlete with a complete intra-substance popliteus tendon rupture, diagnosed on magnetic resonance imaging (MRI) and successfully managed

Received September 26, 2017; Revised (1st) January 22, 2018;

(2nd) March 13, 2018; Accepted April 5, 2018

Correspondence to: Denis Pak-Han Koong, MD, BMed

Department of Orthopaedic Surgery, Royal North Shore Hospital,

3C Clinical Administration Reserve Road, St. Leonards, NSW 2065,

Australia

Tel: +61-4-3344-1221

E-mail: denis.koong@gmail.com

This is an Open Access article distributed under the terms of the Creative Commons Attribution Non-Commercial License (http://creativecommons.org/licenses/by-nc/4.0/) which permits unrestricted non-commercial use, distribution, and reproduction in any medium, provided the original work is properly cited. non-operatively.

\section{Case Report}

A 22-year-old professional rugby league player sustained a lateral blow to his fixed, semi-flexed left knee after being tackled by an opposing player whilst running with the ball. He noted immediate posterolateral knee pain and was unable to play on.

Examination revealed a normal gait, slight varus alignment and a minor knee effusion with tenderness to palpation along the posterolateral joint line. He lacked the terminal $5^{\circ}$ of both flexion and extension range. All ligaments were stable, including the posterolateral corner (PLC), and patella tracking was normal, with no apprehension. Repeat examination 2 days later was similar, with no detectable instability in any plane. X-rays were unremarkable.

An MRI scan was performed, and a complete tear of the popliteus tendon at its musculo-tendinous junction with retraction to the popliteus hiatus and associated oedema around the muscle belly was demonstrated on standard sagittal (Fig. 1) and coronal/ axial views (Fig. 2). The tendon was indistinct at the joint line. No associated injuries of the lateral collateral ligament, posterior 

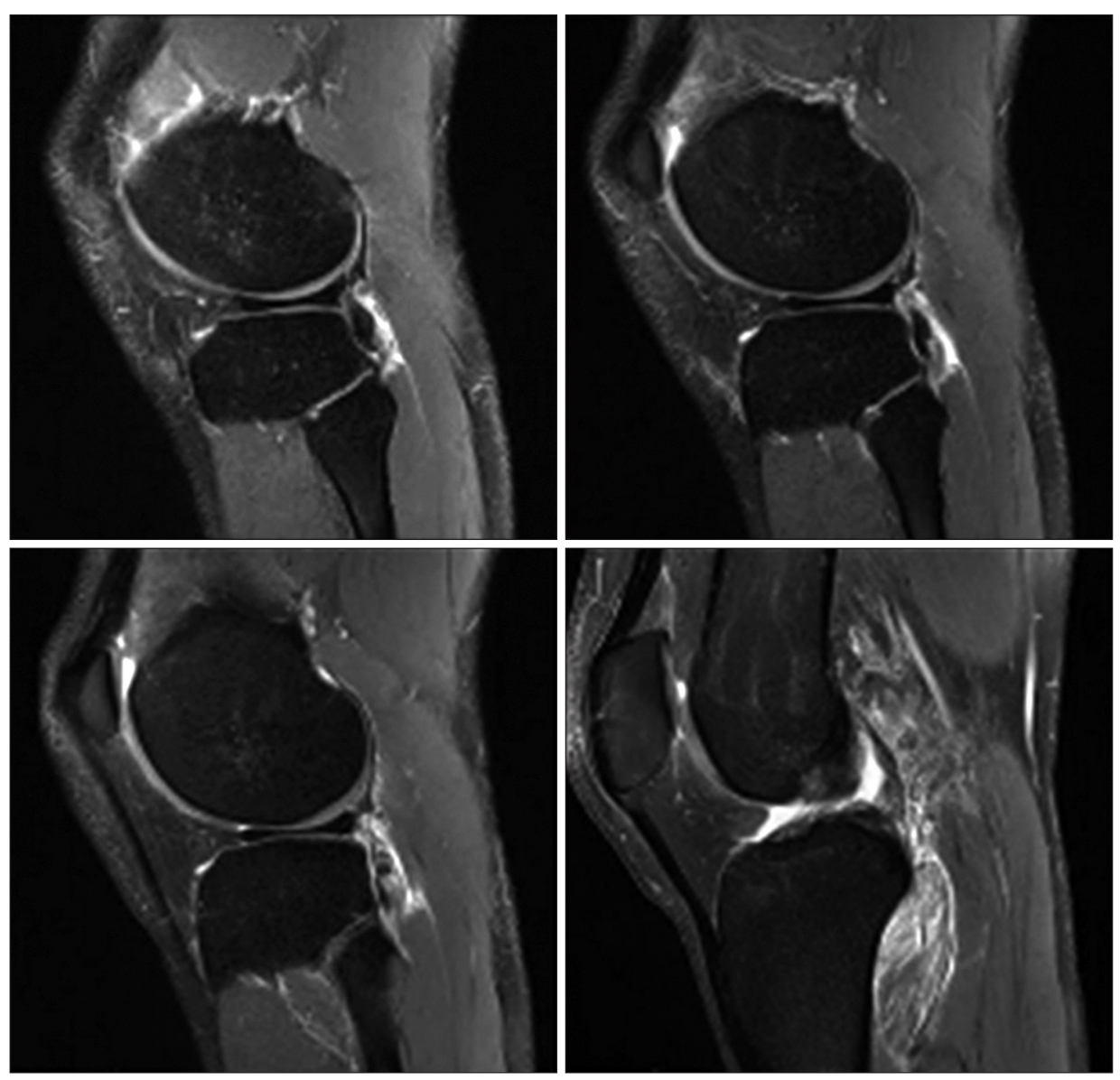

Fig. 1. Sagittal T2-weighted magnetic resonance imaging slices demonstrating significant oedema within the popliteus muscle belly, with indistinct contour at the musculo-tendinous junction indicating an acute injury.
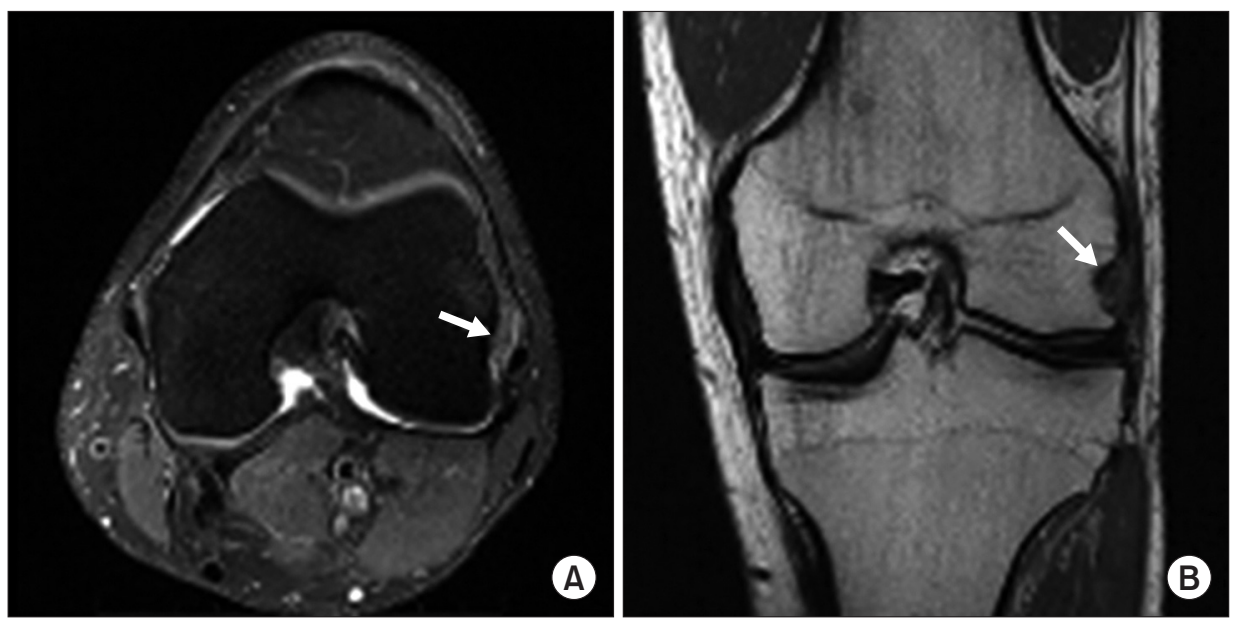

Fig. 2. Axial T2-weighted (A) and coronal T1-weighted (B) slices showing complete avulsion of the popliteus tendon from femoral insertion (arrows), with retraction to the popliteus hiatus.

cruciate ligament or capsule were reported (Figs. 3 and 4). After 4 days of rest and physical therapy, isometric quadriceps exercises including contractions at 5 second intervals and straight leg raises were commenced under the guidance of a physiotherapist. At 1 week, this progressed to weighted straight leg raises and band-assisted exercises targeting the posterior hamstrings and gastrocnemius-soleus complex along with stretching to achieve full range of motion (ROM). Gravity induced posterior sag, and flexion exercises were avoided. Loading of the knee was progressively introduced in the way of quadriceps wall sits and then wall squats. The patient had a graduated return to activity, with participation in running sessions from day 14 and successful return 


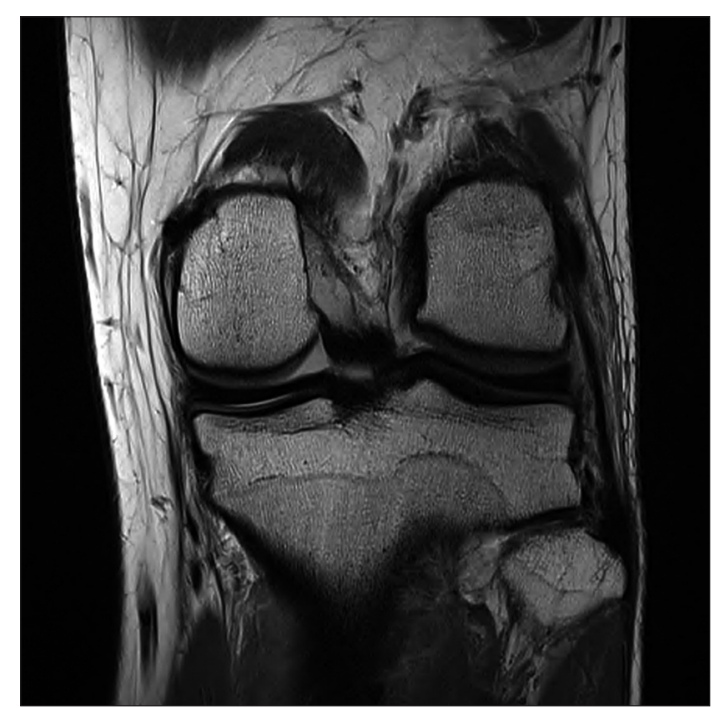

Fig. 3. Coronal T1-weighted image demonstrating the intact lateral collateral ligament.

to full contact within 4 weeks. Follow-up at 15 months revealed a pain-free and clinically stable knee with full ROM.

\section{Discussion}

The popliteus is a triangular muscle that originates on the posteromedial aspect of the tibial metaphysis, proximal to the soleal line, forming the floor of the popliteal fossa. It continues superolaterally as the popliteus tendon, coursing extra-synovially through the popliteal hiatus, and inserts into the outer aspect of the lateral femoral condyle ${ }^{1)}$. The popliteus tendon is strongly anchored to the fibular head with other variable attachments to the posterior horn of the lateral meniscus ${ }^{2,5}$. Dynamically, the popliteus musculo-tendinous unit "unlocks" the knee joint in its weight bearing state and acts as a restraint to forward displacement of the femur on the tibia during initial flexion. In addition, it is important in maintaining static stability of the PLC and lateral knee in cooperation with other principal structures such as the popliteofibular ligament and lateral collateral ligament ${ }^{1,22}$.

Lesions of the popliteus tendon have been attributed to sudden non-contact external rotation of the tibia in a partially flexed knee or forced external rotation with application of varus force with a fixed femur. However, the exact mechanism of injury is often unclear ${ }^{3-6}$. In our case, the mechanism was likely due to a combination of tibial external rotation and the lateral driving force of the opposing tackling player on our patient's flexed knee.

Findings on physical examination are subtle and non-specific. Often, as in our patient, there is a variable joint effusion with

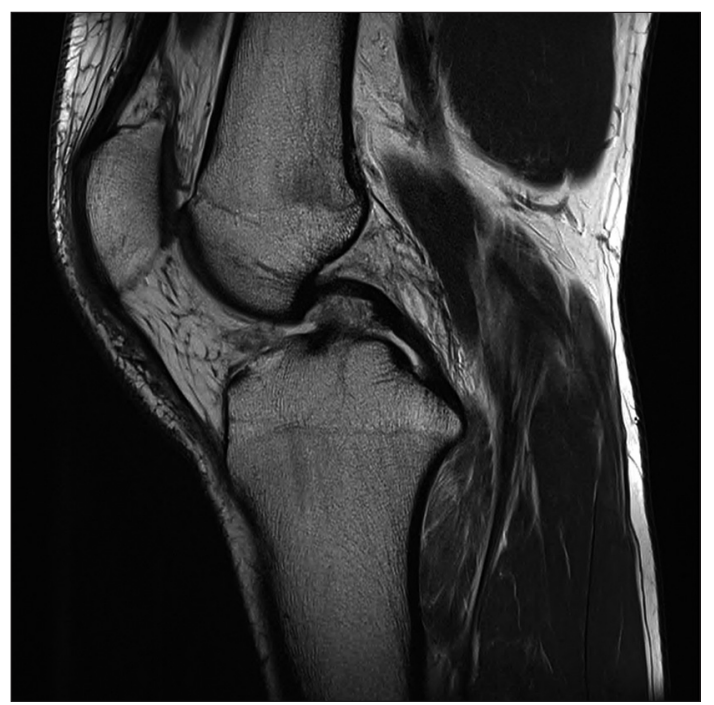

Fig. 4. Sagittal T1-weighted image demonstrating the intact posterior cruciate ligament.

posterolateral joint-line tenderness and mild discomfort on range of movement in an otherwise stable knee ${ }^{5-8)}$. An acute haemarthrosis, posterolateral tenderness in a stable knee and reproducible pain over the popliteus by either passive external rotation or resisted internal rotation of the knee should raise suspicion of an isolated popliteal tendon injury ${ }^{5,6)}$. MRI is a useful modality to evaluate a suspected injury of the popliteus musculo-tendinous unit and exclude commonly associated damage to PLC structures and menisci ${ }^{1)}$. The diagnosis can be confirmed via arthroscopic examination of the lateral gutter and popliteal recess ${ }^{6,7}$.

Popliteus musculo-tendinous ruptures commonly occur in association with other PLC ligamentous injuries ${ }^{1,5)}$. Although surgical reconstruction is often necessary to prevent acute or chronic instability in these situations ${ }^{2,4)}$, surgical repair of isolated popliteus tendon ruptures remains contentious. A significant proportion of popliteus tendon injuries treated operatively are associated with an avulsed osteochondral fragment from its femoral attachment. These were usually suitable for reattachment with a combination of screws, staples or suture anchors, either arthroscopically or using an open approach ${ }^{3,8}$. Rose and Parisien ${ }^{4)}$ did, however, present a case of an intra-substance rupture after indirect trauma that was successfully repaired with non-absorbable sutures.

Nevertheless, in the setting of isolated popliteus tendon ruptures, conservative management with early weight bearing and functional rehabilitation has been demonstrated to be a viable option with similar functional outcomes especially as ligamentous instability is often absent or minimal, ${ }^{5,6)}$. Guha et al. ${ }^{5)}$ reported return to full activity at 6 weeks and no instability at 12 months in a 
semi-professional athlete with a complete intra-substance rupture managed non-operatively, while Burstein and Fisher presented a professional soccer player with an avulsion of the popliteus tendon who successfully regained full function after 4 weeks of rehabilitation with no posterolateral instability at 3-month follow-up. The presence of complementary stabilizers of the PLC may act to maintain overall stability ${ }^{2}$ and therefore, anatomic reduction via surgical repair of isolated popliteal tendon ruptures may not confer significant additional benefit.

There is scarce literature outlining specific rehabilitation protocols tailored for isolated popliteus musculo-tendinous injuries, but in general, non-operative management has involved some combination of a period of rest, immobilisation, strengthening exercises and graduated ROM and activity ${ }^{5,6)}$. One study by Quinlan et al. ${ }^{9)}$ described a detailed rehabilitation program encompassing cable medial rotation strengthening of the knee in varying degrees of flexion, hamstring exercises, dynamic and static proprioception training and progressive activity involving straight-line, agility and resisted running, with a subsequent return to full contact work 5 weeks post injury. Additionally, numerous structured non-operative protocols exist for treatment of grade I and II PLC knee injuries which, in theory, may also be suitably applied to popliteus injuries as it is a key posterolateral structure. These involved immobilisation in a hinged knee brace locked in extension for 2-4 weeks with early initiation of isometric quadriceps exercises followed by progressive ROM exercises, weight bearing and strengthening with return to full activity in 6-12 weeks ${ }^{10)}$.

In comparison, our patient was successfully rehabilitated after a short period of rest with a tailored program that focused on maintaining quadriceps strength and secondary stabilisation with isometric exercises complimented by band-assisted exercises and stretching targeting posterior structures including the gastrocnemius-soleus and hamstring musculature to regain full range of motion within the first 2 weeks. Loading of the knee and graduated activity was progressively introduced during the next phase of our protocol, resulting in return to professional contact sport at 4 weeks post injury. The vast majority of isolated popliteus tendon ruptures treated non-operatively in the literature were sustained during non-contact activities ${ }^{5,6)}$, contrasting the direct contact mechanism experienced by this rugby player. It is interesting to note that these injuries can be successfully rehabilitated in this setting-indeed, our patient continued to be pain-free and playing at professional level at the 15-month follow-up.
Current management of isolated popliteus tendon rupture should ideally be tailored to individual circumstances; with accurate assessment of the extent of injury, integrity of associated structures and patient factors. Furthermore, additional research is needed to evaluate the effectiveness of specific non-operative rehabilitation programs and long-term outcomes of these injuries.

\section{Conflict of Interest}

No potential conflict of interest relevant to this article was reported.

\section{References}

1. Jadhav SP, More SR, Riascos RF, Lemos DF, Swischuk LE. Comprehensive review of the anatomy, function, and imaging of the popliteus and associated pathologic conditions. Radiographics. 2014;34:496-513.

2. Ullrich K, Krudwig WK, Witzel U. Posterolateral aspect and stability of the knee joint. I. Anatomy and function of the popliteus muscle-tendon unit: an anatomical and biomechanical study. Knee Surg Sports Traumatol Arthrosc. 2002; 10:86-90.

3. Westrich GH, Hannafin JA, Potter HG. Isolated rupture and repair of the popliteus tendon. Arthroscopy. 1995;11:628-32.

4. Rose DJ, Parisien JS. Popliteus tendon rupture: case report and review of the literature. Clin Orthop Relat Res. 1988; (226):113-7.

5. Guha AR, Gorgees KA, Walker DI. Popliteus tendon rupture: a case report and review of the literature. Br J Sports Med. 2003;37:358-60.

6. Burstein DB, Fischer DA. Isolated rupture of the popliteus tendon in a professional athlete. Arthroscopy. 1990;6:238-41.

7. Mariani PP, Margheritini F. Partial isolated rupture of the popliteus tendon in a professional soccer player: a case report. Sports Med Arthrosc Rehabil Ther Technol. 2009;1:18.

8. Nakhostine M, Perko M, Cross M. Isolated avulsion of the popliteus tendon. J Bone Joint Surg Br. 1995;77:242-4.

9. Quinlan JF, Webb S, McDonald K, Meikle G, McMahon SF. Isolated popliteus rupture at the musculo-tendinous junction. J Knee Surg. 2011;24:137-40.

10. Rue JP, Kilcoyne K, Dickens J, Kluk M. Diagnosis and treatment of injuries to the posterolateral ligament complex. J Knee Surg. 2011;24:143-50. 